\title{
Apuntes históricos del desarrollo de la normalización del cemento en la Unión Europea
}

JOSÉ CALLEJA

IECA

Fecha de recepción: 3-VI-02

Fecha de aceptación: 10-VI-02

ESPAÑA

\section{RESUMEN}

Al hacerse de obligado cumplimiento en todos los países de la UE a partir del 1 de abril de 2002 la Norma Europea EN 197-1:2000 para cementos, elaborada por el CEN/TC 51, se ha considerado oportuno dar una información general, muy resumida, acerca del desarrollo histórico de las etapas, vicisitudes y dificultades por las que ha pasado la culminación de la misma, hasta alcanzar, primero, la Norma Experimental ENV 197-1:92 y, finalmente, la actual y definitiva EN 197-1:2000, ya en vigor como Norma Española UNE-EN 197-1:2000, de AENOR (1).

\section{SUMMARY}

The European Standard EN 197-1:2000 for Cement, prepared by CEN/TC 51, is form now (2002-04-01) onwards, compulsory in all the countries of the European Union. It has been considered suitable to expose some details on the historical development of the steps and difficulties during the elaboration of the standard ENV 197-1:92 first, and of the present and final standard EN 197-1:2000 already in force, and now in Spain as Spanish Standard AENOR/UNE-EN 197-1:2000(1).

\section{INTRODUCCIÓN}

La Norma Europea para Cementos EN 197-1: 2000 es ya de obligado cumplimiento, a partir del 1 de abril de 2002, en sustitución de todas las normas nacionales homólogas de los países de la UE que no estén en consonancia con ella.

La consecución de esta meta no ha sido fácil. Ha exigido recorrer lentamente un largo camino lleno de obstáculos difíciles de vencer, y ello a un alto costo de trabajo-tiempo y también de dinero.

No obstante, no cabe pensar que la Norma es una obra completa e inmodificable, ya que a lo largo de su aplicación pudicra ser objeto de cambios, ajustes o adendas aconsejados por la experiencia y el conocimiento que de la aplicación de la misma se vayan adquiriendo.

Es, por lo tanto, una norma perfectible, máxime contando con el muy probable y próximo ingreso en la UE de otros países de Europa Central y Oriental, los cuales, en su momento, aportarán sus ideas, sugerencias y propuestas a la Normalización Europea de Cementos.

En lo que sigue se da cuenta de las fases y etapas del desarrollo de esta normalización, que ya tiene historia, dentro de la UE y en lo que respecta a España.

\section{ANTECEDENTES}

Hace ahora ya más de medio siglo que, al final de la Segunda Guerra Mundial, concluida en 1945, el Premier británico Sir Winston Churchill propuso la creación de unos "Estados Unidos de Europa".

En 1950 el Canciller alemán Robert Schuman impulsó la creación de la CECA (Comunidad Europea del Carbón y del Acero), la cual quedó establecida en 1951, fecha en la que se firmó el Tratado de París.

Nacida la unidad política de Europa y, tras el fracaso de una Comunidad Europea de Defensa, se avanzó por el flanco de lo económico, y así, en 1957 se suscribieron los 
Tratados de Roma, por los cuales se crearon la CEE (Comunidad Económica Europea) y la CEEA (Comunidad Europca de la Energía Atómica), también conocida como el "EURATOM". Así se cumplió la primera etapa de la carrera europea.

Hasta 1969 y en adelante se fucron desarrollando en el seno de la CEE diferentes organismos, de modo que en 1973 se creó el Comité Europeo de Normalización, CEN, para llevar a cabo la relativa a todos los productos normalizables. Dentro del mismo se estableció toda una serie de Comités Técnicos, entre ellos el TC 51: "Cementos y Cales de Construcción", previsto para elaborar las normas de dichos productos o materiales en los países de la CEE y en los de la Asociación Europea de Libre Comercio (EFTA: European Free Trade Association).

Entretanto, en 1972 ingresaron en la CEE el Reino Unido, Irlanda y Dinamarca, después Grecia en 1981, y finalmente España y Portugal en 1985.

En 1986 se promulgó la llamada Acta Única, firmada el año anterior, por la cual se estableció el Mercado Común sin fronteras, previsto para entrar en vigor en 1993, con la libre circulación de productos y mercancías, sin trabas aduaneras, en todos los países miembros de la Comunidad Europea (CE).

Bastante antes, a partir de 1973, comenzaron formalmente las tareas de la normalización del cemento, las cuales se han venido desarrollando de modo prácticamente ininterrumpido, en particular durante los años 80 , en los cuales el CEN/TC 51 decidió tener en cuenta, a efectos de su inclusión en un Proyecto de Norma Europea Experimental -"prenorma"-, (prENV 197-1), sólo los "cementos comunes", entendiendo por tales los utilizables en hormigón en masa, armado y pretensado, producidos y utilizados en la mayoría de los países de la Europa Occidental. Este propósito incluía también todo lo relativo a la definición, clasificación, nomenclatura-denominación y designación- y especificaciones de los cementos, así como lo tocante a los métodos de ensayo y análisis de los mismos.

Fue así, como en 1989 el CEN/TC 51, a través de sus Subcomités y Grupos de Trabajo culminó la elaboración de un proyecto de norma europea para cemento, el cual, propuesto y sometido a votación, fue rechazado el mismo año por falta de aceptación mayoritaria, y porque la $\mathrm{Di}$ rectiva de Productos de la Construcción de la CE previamente promulgada y que, naturalmente afectaba al cemento, exigía la inclusión en el Proyecto de todos los cementos tradicionales y bien experimentados en todos los países de la Comunidad, a fin de establecer el ya citado Mcrcado Común Europeo para dichos productos, previsto en la mencionada Acta Única; exigencia que cl proyecto presentado no cumplía.
La Directiva 89/106/CEE, prevista como otras en el Artículo 100 del Tratado de Roma -Artículo 100 A del Acta Única-, nacida en diciembre de 1988 y publicada en el Diario Oficial de las Comunidades Europeas en febrero de 1989, la cual se consolidó después como vigente, establecía asimismo criterios a seguir, no sólo para la normalización, sino también para la certificación en el campo de los productos de la construcción, ateniéndose a la Resolución del Consejo, de mayo de 1985, relativa a un "Nucvo Enfoque" en materia de armonización técnica y normalización.

La Directiva fijaba también un plazo de 30 meses, hasta Junio de 1991, para ser cumplimentada por los Estados Miembros. Y, por supuesto, como toda otra Directiva de la $C E$, hubo de ser transferida a la legislación nacional de cada Estado Miembro para poder ser aplicada en todos ellos.

El proyecto prENV 197-1, después de algunos retoques y previas votaciones preliminares y fïnal, fue publicado en 1992 como "norma experimental", con la designación ENV 197-1:92; fecha a partir de la cual sc produjo un cierto estancamiento en cl avance de esta norma.

El hecho es que entre 1973 y 1992 -y eso sin tener en cuenta que con anterioridad a la primera de dichas fechas había ya antecedentes de normalización desde 1969, no coordinados ni adscritos todavía al Comité CEN/TC 51, tuvieron que pasar nada menos que 20 años para que hubiera un proyecto de norma curopea para cementos; eso si, "experimental" -esto es, provisional, transitoria, no definitiva-.

¿Ha supuesto esto incapacidad por parte de los expertos y especialistas de los 12 (15) países de la CE primero (y de la UE después) para elaborar el proyecto prENV 1971:92 y, andando el tiempo, la norma final EN 197-1:2000, a la cual se ha llegado al cabo de 30 años- ¡casi un tercio de siglo!-? Evidentemente no, puesto que cuando han tratado de elaborar sus respectivas normas nacionales -y algunos casos se han dado durante el mencionado período de tiempo-, las han elaborado fácil y rápidamente, y a gusto y satisfacción de fabricantes y usuarios del cemento, en cada país y circunstancia.

Si en cl caso de la norma curopea no ha sido así, la razón hay que buscarla -y encontrarla- en un trasfondo de intereses enfrentados, ciertamente que no técnicos, entre unos y otros países con diferentes características, circunstancias y puntos de vista.

Por otra parte, en 1992, fecha como se ha visto clave en el camino de la normalización europea de cementos, se empezó a considerar la necesidad urgente de elaborar un Mandato de Normalización para los mismos, hasta entonces sorprendentemente no redactado ni, por lo tanto, 
publicado, a pesar de que el CEN/TC 51 ya llevaba los años dichos trabajando en la normalización del cemento. Y tanto más, cuanto que otros productos de normalización muy incipiente, incluso aún no comenzada, disponían ya, de antemano, de su Mandato correspondiente.

También en 1992 se preparó y firmo cl Tratado de la Unión Europea (UE), llamado de Maastricht, cuya primera revisión se previó para 1996. Según las disposiciones del mismo la Unión Europea pasaría a tener una serie de Instituciones y de competencias Políticas, de Presupuesto, de Derecho y de Moneda comunes y propias. Entre las Instituciones figuraban el Parlamento, el Consejo, la Comisión y el Tribunal del Justicia. Entre las competencias Políticas destacaban las relativas a la Agricultura, la Unión Aduanera, el Transporte, la Comercial y la de la Libre Competencia. El Presupuesto sería común; y el Derecho, propio. En cuanto a la Moneda Única, el "ECU" -entonces-, estaba previsto para 1997 ó 1999. Su sucesor, el "EURO", ya lo tenemos en las manos, eso sí, retrasado al 2002.

Fue en 1997 cuando tuvo lugar en Ámsterdam la negociación de la Conferencia Intergubernamental para la Reforma de los Tratados -entre ellos cl de Maastricht, al cual le llegó con un año de retraso con arreglo a lo previsto-; Conferencia que se produjo tras dos años de largos debates sobre Política Exterior y Común de la UE, Equilibrio entre Estados Miembros en el Consejo de Europa, Composición de la Comisión Europea y Evolución hacía la Moneda Única, objetivos en gran parte ya logrados.

Estos han sido los antecedentes y las etapas a lo largo de las cuales se llegó desde un definido proyecto de norma prENV 197-1, a la Norma Europea Experimental ENV 197-1:92, base de partida de la actual, al fin ya lograda, EN 197-1:2000, transferida a la española UNE-EN 1971:2000 de AENOR (1).

\section{CONSECUENTES}

La norma "experimental" ENV 197-1 de 1992 incluía, como queda dicho, solamente los llamados "cementos comunes", ya definidos.

Por otro lado, el CEN/TC 51, en buena lógica, y dado el elevado número de tipos de cementos "comunes y no comunes" -entendiendo por éstos últimos los dotados de alguna característica adicional especial, o uso o aplicación específica, no imputables a los "comunes"-, decidió dividir cl Proyecto ENV 197 en distintas partes, la primera de las cuales (ENV 197-1) incluía sólo los cementos cuyo endurecimiento se basa principalmente en la hidratación de los silicatos de calcio anhidros del clínker portland.

Otras partes sucesivas irían incluyendo otros tipos de ccmento con otros mecanismos diferentes de endurecimiento -por ejemplo, los después llamados "ccmentos de aluminato de calcio" y antes "cementos aluminosos"-; o con alguna propicdad o comportamiento específico añadido como resistencia a los sulfatos y/o al agua de mar, bajo calor de hidratación, bajo contenido de álcalis, color blanco..., en una gama muy amplia (entonces) de posibilidades-.

La Norma Europea Experimental ENV 197-1:92 estaba previsto que al cabo de tres años a partir de 1992 y tras dicho período de adaptación en paralelo y simultaneidad con las nacionales de los Países Miembros de la UE, fuese aceptada y adoptada por los mismos, convirtiéndose en norma final y definitiva EN 197-1; o, que en otro caso, pasase a ser revisada de manera que tras otro período de revisión de dos años más, y con las modificaciones introducidas en ella en virtud de dicha revisión, pudicra ser aceptada como EN 197-1; o, en caso contrario, retirada. En tal situación extrema la Comisión de la Comunidad Europea (CCE) tomaría por sí misma una decisión inapelable al respecto.

Durante el primer período de tres años (1992-1995) estaba prevista la traducción del texto matriz de la ENV $197-$ 1 a los respectivos idiomas de los distintos países de la UE para su conocimiento y difusión, por parte los Organismos Nacionales de Normalización-AENOR, en el caso de España- y para los Sectores Fabricantes y Usuarios de los cementos, Y también para la aplicación y puesta en práctica de dicha norma, con la consiguiente anotación de posibles fallos, defectos o aspectos de la misma dignos de discusión y posible corrección, en su caso. Esto fue puntualmente llevado a cabo.

En 1995 la norma ENV 197-1:92 no fue aceptada como EN 197-1 definitiva, a consecuencia del voto negativo de Alemania y de la abstención del Reino Unido en la votación.

El rechazo alemán, paradójicamente al hecho de que el Instituto Alemán de Investigación de la Industria del Cemento (Forschungsinstitut der Zementindustrie) de la Agrupación de Fábricas Alemanas de Cemento (Verein Deutscher Zementwerke -VDZ-) en Dusseldorf. había venido ostentando la presidencia del Grupo de Trabajo WG 6 del CEN/TC 51, grupo precisamente encargado de la promoción y avance de la norma, se basó en que, según la opinión alemana, los cementos incluidos en ella "no eran adecuados para cada propósito de utilización de los mismos". Dicha opinión estimaba asimismo que en la norma ENV 197-1 faltaban criterios que permitieran determinar cuáles de los 25 tipos de cementos posibles incluidos en ella entonces -hoy son, como se sabe, 27- cran adecuados, por una parte, para obtener hormigón resistente a las heladas; $y$, por otra parte, para garantizar la protección de las armaduras del hormigón contra la corrosión. Se adujo también que estas "insuficiencias" de la ENV 197-1 no se atenuaban con la Tabla 3 de la Norma 
Experimental para Hormigones ENV 206 (del Comité CEN/TC 104), en la cual se señalaban los requisitos para los hormigones en cuanto a su durabilidad en condiciones ambientales de exposición, las cuales, a su vez, se especificaban en la Tabla 2 de dicha norma. Esto era, cuando menos, discutible, y probablemente, poco sostenible.

En definitiva, el voto negativo alemán se basó en la no existencia, por el momento, de los citados criterios, así como de métodos de ensayo adecuados para garantizar el comportamiento y prestaciones ("performances") de determinados cementos de "nuevo cuño" -para los alemanes-, acerca de los cuales la experiencia previa se suponía o se daba por sentado -por parte de los alemanes, claro- que era escasa o inexistente en cuanto a las mencionadas resistencias al hiclo y protección de las armaduras se refería. Todo ello, sc insiste, según cl discutible criterio alemán.

La abstención del Reino Unido se basó en que en la Norma Experimental ENV 197-1 no estaban adecuadamente considerados aspectos relativos a la salud y a la seguridad, particularmente por lo que se refería a la insuficiente garantía para evitar la contaminación de aguas potables por compuestos tóxicos solubles de metales pesados procedentes de cementos que contuviesen determinadas "puzolanas industriales", entonces definidas como escorias de metalurgias del plomo, cobre, níquel, cinc, etc., distintas de la siderurgia, designadas por "Q".

Hay que señalar que dicha abstención se produjo por un solo voto en contra del Comité de Normalización de Cemento de la BSI (British Standard Institution), el organismo inglés de normalización, en el cual es exigible la una- nimidad, según sus propios reglamentos. La argumentación expuesta por España en su momento, en contra del mencionado escrúpulo, había sido aceptada por todos los demás miembros de dicho Comité, excepto por el citado único miembro discrepante. Así, pues, la abstención británica tuvo el carácter de un verdadero veto que, junto con la actitud alemana, supuso un nucvo retraso en el avance de la norma, y que obligó a desarrollar un nuevo plan de trabajo por parte del CEN/TC 51, a partir de entonces, y programado como sigue.

Desde mayo de 1995 comenzó la preparación del primer borrador del proyecto de norma final prEN 197-1, el cual debcría estar concluído en 1996 y ser cnviado a cncuesta pública para, en mayo de 1997, ser tomado en consideración, habiéndose fijado el plazo entre 1997 y 1998 para la emisión del voto formal sobre dicho proyecto; y entre 1998 y 1999 para la implantación del mismo, una vez convertido en norma europea definitiva EN 197-1:99.

¿Se han cumplido dichas etapas y plazos? Evidentemente, no. En 1997 se estaba al término del último plazo de los dos años para que el proyecto prEN 197-1, que se había venido elaborando sobre la base de la norma experimental rechazada, fuese finalmente aceptado como EN 197-1 final o, por el contrario, fuese definitivamente retirado.

El futuro de entonces es ya la realidad de hoy, con la Norma Europea para Cementos EN 197-1:2000, a la cual se ha llegado a través de la evolución del proyecto hasta acabar en la norma que, como queda dicho es ya la española UNE-EN 197-1:2000 de AENOR, vigente desde el 1 de abril de 2002.

\section{BIBLIOGRAFÍA}

(1) J. CALLEJA: "La entrada en vigor de la Norma UNE-EN 197-1:2000 para Cementos en la Unión Europea". CEMENTO-HORMIGÓN, Núm. 835, abril 2002, págs. 4-19. 